\title{
Infrared electron modes in light deformed clusters
}

\author{
V.O. Nesterenko ${ }^{1,2,5}$, P.-G. Reinhard ${ }^{3}$, W. Kleinig ${ }^{1,4}$, and D.S. Dolci ${ }^{1}$ \\ ${ }^{1}$ BLTP, Joint Institute for Nuclear Research, Dubna, Moscow region, 141980, Russia \\ ${ }^{2}$ Max Planck Institute for Physics of Complex Systems, D-01187, Dresden, Germany \\ ${ }^{3}$ Institut für Theoretische Physik, Universitat Erlangen, D-91058, Erlangen, Germany \\ ${ }^{4}$ Technische Univirsitat Dresden, Inst. für Analysis, D-01062, Dresden, Germany and \\ 5 E-mail: nester@thsun1.jinr.ru
}

\begin{abstract}
Infrared quadrupole modes (IRQM) of the valence electrons in light deformed sodium clusters are studied by means of the time-dependent local-density approximation (TDLDA). IRQM are classified by angular momentum components $\lambda \mu=20,21$ and 22 whose $\mu$ branches are separated by cluster deformation. In light clusters with a low spectral density, IRQM are unambiguously related to specific electron-hole excitations, thus giving access to the single-electron spectrum near the Fermi surface (HOMO-LUMO region). Most of IRQM are determined by cluster deformation and so can serve as a sensitive probe of the deformation effects in the mean field. The IRQM branch $\lambda \mu=21$ is coupled with the magnetic scissors mode, which gives a chance to detect the latter. We discuss two-photon processes, Raman scattering (RS), stimulated emission pumping (SEP), and stimulated adiabatic Raman passage (STIRAP), as the relevant tools to observe IRQM. A new method to detect the IRQM population in clusters is proposed.
\end{abstract}

\section{INTRODUCTION}

The most prominent electron mode in metal clusters is the Mie surface plasmon [1]. Being the doorway to many structural and dynamical properties, it has been much investigated in the past, for extensive summaries see e.g. 2, [3, 4, 5]. Besides the dominant Mie plasmon, many other electron modes, both electric and magnetic, have been predicted but not yet observed in clusters, for a brief overview see [6]. Similar modes exist in other manyfermion systems. In particular, almost all of them were identified experimentally in atomic nuclei, for a review see 7].

The analogy with deformed nuclei suggests that deformed clusters should exhibit a family of low-energy (infrared) electron modes which are absent in spherical systems. Deformed clusters have a partly filled valence electron shell and so sustain low-energy one-electron-onehole (1eh) excitations inside this shell. Being mainly determined by the deformation splitting, the excitation energies are rather small and typically lie in the infrared. Since major shells in light clusters cover electron levels with the same space parity, the infrared modes should have positive parity. Their softness and deformation dependence suggest that these should be infrared quadrupole modes (IRQM). In axially deformed clusters, IRQM are represented by separate $\lambda \mu=20,21$ and 22 branches (where $\lambda=2$ stands for total angular momentum and $\mu$ its azimuthal component).

Our present knowledge on infrared electronic excitations in clusters is very poor. Even basic IRQM properties (spectrum, collectivity, evolution with cluster size and deformation, responses to external fields) are unknown. It is the aim of this paper to investigate main features of IRQM from a theoretical perspective, hopefully delivering useful hints for an experimental search.

IRQM in light and in heavy clusters have a different origin [8]. In the present paper, we will consider light clusters or, specifically, free deformed singly-charged light clusters. Such clusters have essential advantages with respect to IRQM. First, they have an extremely low spectral density at low excitation energies, a feature which facilitates a discrimination of IRQM in twophoton processes. The low spectral density also reduces the unwanted level broadening (Landau fragmentation, electron-electron collisions). Beams of size-selected singly-charged light clusters are readily available. So, these clusters are well suited for measurements. Second, as is shown below, IRQM in light clusters are dominated by one 1eh component and can be directly and unambiguously interpreted in terms of the electron spectrum in the HOMO-LUMO region. So, IRQM can deliver an important information on the underlying mean field level structure. Since most of IRQM are induced by cluster deformation, they allow to study the evolution of the electronic levels with deformation. And third, there is a close connection between $\lambda \mu=21$ branch of the IRQM and the electronic scissors mode (SM) 9, 10, 11, 12. This gives a chance to observe the SM, at least indirectly, through IRQM. SM is a universal dipole magnetic orbital mode peculiar to deformed systems. It was already observed in a variety of quantum systems but not yet in clusters.

Our calculations were performed within a linearized time-dependent local density approximation (TDLDA). The ionic background is approximated by a soft deformed jellium density, for reviews see [13, 14]. The method goes back to early studies on cluster spectra 15]. It has been successfully applied to study the dipole plasmon in spherical [16] and deformed [17, 18] clusters. We will consider here Na clusters as the simplest test case. However, IRQM properties worked out below are of a general character and so should appear for all other metal clusters with pronounced shell structure for valence electrons.

IRQM are not accessible by one-photon transitions which excite exclusively dipole modes. Thus one has to use two-photon processes (TPP) where the target state is 
populated via an intermediate state by two (absorption and emission) dipole transitions. The dipole plasmon can serve as an intermediate state.

Experimental data on IRQM are very scarce and limited to heavy clusters (e.g. RS measurements of IRQM in embedded silver clusters were reported [19]). Moreover, typical two-photon techniques of atomic and molecular spectroscopy are rarely used for clusters. This can be partly explained by the fact that clusters have some specific properties (see discussion in Sec $\nabla$, which makes a straightforward application of these techniques questionable. In particular, the common methods of detection of the level population, based on the radiative decay, seem not to be appropriate for clusters. In this paper, we will examine applicability to clusters of some widespread TPP: Raman scattering (RS), stimulated emission pumping (SEP), or stimulated Raman adiabatic passage (STIRAP). An alternative method for detection of the level population in clusters will be proposed.

Altogether, the present study has two goals: i) to provide a first guide on quadrupole electronic excitations in light deformed clusters; ii) to explore the access to nondipole electron modes in clusters by modern two-photon techniques.

The paper is outlined as follows. Section $\llbracket$ describes the theoretical framework and choice of the most suitable clusters. In section \III a general hierarchy of quadrupole electron excitations in clusters is presented and the origin of IRQM is clarified. In section IV the IRQM responses in one- and two-photon processes are compared and the 1eh nature of IRQM is demonstrated. In section $\nabla$ the possibility to observe IRQM in two-photon process (RS, SEP and STIRAP) is discussed. Conclusions are drawn in section VI In Appendix A, the dipole matrix elements responsible for the coupling between the states involved into two-photon process are analyzed. In Appendix B, the scissor mode and its connection with the $\lambda \mu=21$ branch of the IRQM are briefly discussed.

\section{CALCULATION SCHEME AND CLUSTER CHOICE}

\section{A. Basic spectral properties}

The electron cloud is described by the densityfunctional theory at the level of the local-density approximation (LDA) for the ground state and time-dependent LDA (TDLDA) for the excitations, using actually the functional of [20]. The ionic background of the cluster is approximated by the soft jellium model allowing for quadrupole and hexadecapole deformation [21]. IRQM stay in the regime of small amplitudes. We thus employ linearized TDLDA, often called the random-phaseapproximation (RPA). The actual implementation in axial symmetry is explained in [17]. The reliability of the method has been checked in diverse studies of the Mie plasmon in spherical [16] and deformed [17, 18] clusters.
The choice of the clusters was dictated by the following reasons: i) The clusters should be small enough to possess a dilute and non-collective IRQM spectrum. Only then the spectrum can be resolved and unambiguously related to the single-particle levels. ii) Since IRQM are mainly induced by cluster deformation, the clusters with a strong deformation (both prolate and oblate) are desirable. The simplest case of axial shape is most suitable for the analysis. iii) Shape isomers exhibit different singleelectron spectra [17, 18], which can result in smearing out the low-energy spectral lines. The heavier are clusters, the more isomers they have [17, 18]. So, the light clusters with a strictly dominant equilibrium shape are preferable. Between them, we should choose the clusters whose ground state and isomers have the similar (prolate or oblate) shape. Thus we will minimize the blurring IRQM spectra. iv) The jellium approximation is not correct for very small clusters. This establishes a lower limit for the cluster size.

The cluster choice can be preliminary done by reviewing the properties of the dipole plasmon which will serve as intermediate state in a two-photon process. Besides, description of the dipole plasmon allows to estimate accuracy of the model. Fig. 1 shows RPA results for the dipole plasmon in light axially deformed Na clusters: prolate $\mathrm{Na}_{11}^{+}, \mathrm{Na}_{15}^{+}, \mathrm{Na}_{27}^{+}$and oblate $\mathrm{Na}_{7}^{+}, \mathrm{Na}_{19}^{+}$. The shape of each cluster is characterized by equilibrium quadrupole and hexadecapole deformations, $\delta_{2}$ and $\delta_{4}$, obtained by minimization of the total cluster energy [17, 18, 21]. To simulate temperature and other sources of line broadening, the photoabsorption cross section is smoothed by a Lorentzian [17].

As is seen from Fig. 1, the emerging dipole spectra consist basically of two peaks, the weaker one $(\lambda \mu=10)$ corresponds to oscillations along the symmetry axis and the stronger one to the orthogonal mode $\lambda \mu=11$ (in fact, two identical modes $\mu= \pm 1$ ). In most of the clusters, these peaks are well separated by the deformation splitting. The most heavy sample $\mathrm{Na}_{27}^{+}$shows some Landau fragmentation of the resonance peaks (distribution of the collective strength between several RPA levels indicated by vertical bars). This is the beginning of a trend which persists towards heavier clusters. The Landau fragmentation overrules the deformation splitting already at $N \approx 50$ [18]. Thus one should stay in the region $N \leq 20$ where the spectra are still dilute and the Landau fragmentation is weak. Within that region, one should pick samples with a large deformation. The clusters $\mathrm{Na}_{7}^{+}, \mathrm{Na}_{11}^{+}$ and $\mathrm{Na}_{15}^{+}$seem to be the best candidates. Besides, their isomeric and ground states have the similar shapes [22], oblate in $\mathrm{Na}_{7}^{+}$and prolate in $\mathrm{Na}_{11}^{+}$and $\mathrm{Na}_{15}^{+}$. The analysis of IRQM in the next section confirms this cluster choice.

Fig. 1 shows that in general the folded spectra (solid lines) agree nicely with the experiment (triangles) which signifies the reliability of the method. However, the jellium plus RPA description, working well for the heavier clusters, worsens for lighter ones. In $\mathrm{Na}_{7}^{+}$and $\mathrm{Na}_{11}^{+}$, the 
calculated spectra are redshifted and do not reproduce the structure details. The discrepancy is due to the fact that light clusters tend to be more compressed due to larger surface tension [24]. But we use here a constant Wigner-Seitz radius for reasons of simplicity. Besides, the jellium approximation is certainly too rough for the description of details in so light clusters. The results for the smallest samples may not reach a quantitative level, but they are still useful for qualitative consideration as will be done here. Fig. 1 shows that even in the lightest clusters the jellium TDLDA sufficiently well reproduces the average energy, principle gross-structure, and magnitude of the deformation splitting of the resonance. Such accuracy suffices for our present survey.

\section{B. Two photon process}

We consider a two-photon process running via $\lambda \mu=10$ or 11 branches of the dipole plasmon as the intermediate states, see Fig. 2. The branches are assumed to be well separated by deformation splitting, which is indeed the case for strongly deformed light clusters (see Fig. 1). Then the two reaction paths can be disentangled by tuning the photon frequency. This allows to specify and monitor the process. For example, if the reaction runs only via $\lambda \mu=10$ plasmon branch, then the population of the low-energy $\lambda \mu=22$ mode is forbidden and only 20 and 21 modes remain to be considered. If one of the paths is suppressed, (see discussion for $\mathrm{Na}_{7}^{+}$in the next section), then the reaction can be tuned to another path. The comparison of the path rates delivers important information about the structure of the dipole plasmon and IRQM.

The population of the IRQM is approximately calculated as a coherent sum of independent two-step processes, each one being a product of dipole photoabsorption and photoemission:

$$
\begin{gathered}
\sigma_{\uparrow \downarrow}\left(2 \mu_{2} i_{2}\right)=\sum_{i_{1}} \sigma_{E 1 \uparrow}^{a b}\left(0 \rightarrow 1 \mu_{1} i_{1}\right) \sigma_{E 1 \downarrow}^{e m}\left(1 \mu_{1} i_{1} \rightarrow 2 \mu_{2} i_{2}\right) \\
\propto \sum_{i_{1}} \omega_{1 \mu_{1} i_{1}}\left|\left\langle 1 \mu_{1} i_{1}|e \mathbf{r}| 0\right\rangle\right|^{2} \\
\cdot\left(\omega_{1 \mu_{1} i_{1}}-\omega_{2 \mu_{2} i_{2}}\right)^{3}\left|\left\langle 2 \mu_{2} i_{2}|e \mathbf{r}| 1 \mu_{1} i_{1}\right\rangle\right|^{2}
\end{gathered}
$$

Here $\mid 1 \mu_{1} i_{1}>$ and $\mid 2 \mu_{2} i_{2}>$ are RPA states of the dipole and quadrupole spectra, respectively. The index $i_{1}$ runs over all RPA states with dipole content in the accessible energy interval. The photoabsorption (photoemission) dipole matrix elements in (1) define the coupling between the ground and dipole (dipole and quadrupole) states in two-photon processes. This coupling determines the Rabi frequency, the decisive value in TPP.

Eq. (11) follows from the general expression for RS rate given elsewhere (see, e.g. [25]) if one keeps only the Stokes term and neglects the interference between the neighboring intermediate states. The Stokes term alone suffices to illustrate the population of IRQM in twophoton processes. Moreover, already the dipole matrix elements in (11) provide a solid ground for the analysis. As for the interference, it should be weak in light clusters. Indeed, in Fig. 1 the dipole plasmon in clusters with $N \leq 20$ is represented by a few states separated by large energy intervals. In any case, the interference mainly leads to smoothing of the response, which can be taken into account by a reasonable averaging the results. In the present paper, we simulate the interference, temperature and other smearing factors by folding the results by a Lorentzian profile with an averaging width $\Delta$. The spectral width is known to increase with the excitation energy. So, we use $\Delta=0.25 \mathrm{eV}$ for the high-energy dipole and quadrupole plasmons (like in our previous RPA calculations for the photoabsorption spectra [16, 17, 18]) and $\Delta=0.1 \mathrm{eV}$ for the low-energy IRQM.

It worth noting that the photoabsorption and photoemission dipole matrix elements in (1) have essentially different structure (see Appendix A for more detail). The former is determined by the 1eh part of the dipole operator $e \mathbf{r}$ and is generally large. The latter is given by $1 e e$ (electron-electron) and $1 \mathrm{hh}$ (hole-hole) parts of the dipole operator and its value can broadly vary, depending on the structure of the dipole and quadrupole states.

\section{IRQM IN THE HIERARCHY OF QUADRUPOLE EXCITATIONS}

The spectrum of the three-dimensional harmonic oscillator provides a useful sorting scheme for the valence electron levels in metal clusters 3 , [4, 26]. The levels are sorted in perfectly degenerate bunches, the major quantum shells characterized by the principle quantum number $\mathcal{N}=0,1,2, \ldots$. The shells are separated by appreciable energy gaps and every shell involves only states of the same space parity $\pi=(-1)^{\mathcal{N}}$. This oscillator picture is well fulfilled in light clusters and provides still a good approximation in medium and heavy ones. In axially deformed clusters, the levels are characterized by Nilsson-Clemenger quantum numbers [26] $\nu=\left[\mathcal{N} n_{z} \Lambda\right]$ where $\mathcal{N}$ is the major shell as before, $n_{z}$ is the number of nodes along the symmetry axis $z$, and $\Lambda$ is the projection of the orbital moment onto the same axis. Following this scheme, excitations of valence electrons are characterized by the $\Delta \mathcal{N}$ value, the difference in shell for the dominant 1eh jumps. Quadrupole excitations have even parity and are collected into the branches $\Delta \mathcal{N}=0$ and 2. The excitations with larger $\Delta \mathcal{N}$ are weak and can be neglected.

Figure 3 illustrates the hierarchy of quadrupole excitations. The lower panels show the unperturbed photoabsorption calculated for the pure $1 \mathrm{eh}$ states ignoring the residual interaction. The upper panels give the RPA photoabsorption. Let us first look at the strong quadrupole resonance appearing at high frequencies in the range $2-4 \mathrm{eV}$. It is blue-shifted by the residual inter- 
action and the larger the cluster, the stronger the shift 29]. In heavy clusters this resonance is associated with the quadrupole plasmon. The resonance is mainly formed by E2 transitions over two major shells $(\Delta \mathcal{N}=2)$. It exists in clusters of any shape and exhausts most of the quadrupole strength. The resonance is energetically very close to the dipole Mie plasmon (in a simple estimate $\omega_{\lambda}=\omega_{\mathrm{pl}} \sqrt{\lambda /(2 \lambda+1)}$ where $\omega_{\mathrm{pl}}$ is the frequency of the volume plasmon [4]). In spite of the energy overlap with the dominant E1 plasmon, the E2 plasmon may, in principle, be discriminated by means of angular-resolved electron energy-loss spectroscopy (AR-EELS) at electron scattering angles $\sim 6^{\circ}[30$.

In the present study, we are interested not in the highenergy E2 resonance but in the IRQM which, being dilute and non-collective, can deliver important information about the electron single-particle spectrum near the Fermi (=HOMO) level. IRQM are associated with the low-energy $\Delta \mathcal{N}=0$ branch created by E2 transitions inside the valence shell. Being of the $\Delta \mathcal{N}=0$ origin, IRQM can exist only in clusters with partly occupied valence shell, i.e. in deformed clusters. In Fig. 3, IRQM reside at $0.5-1.5 \mathrm{eV}$. As compared with the $\mathrm{E} 2$ resonance, the IRQM spectrum is very dilute. It is represented only by a few well separated levels. This prevents mixing of $1 \mathrm{eh}$ configurations by the residual interaction and creation of collective states. The IRQM persist to keep their 1eh nature and so can be easily identified as particular $1 \mathrm{eh}$ configurations. As is seen from Fig. 3, the IRQM have very weak quadrupole strength in the photoabsorption. But they may be accessible in two-photon reactions.

Fig. 4 shows single-particle levels and 1eh quadrupole transitions inside the valence shells in $\mathrm{Na}_{7}^{+}, \mathrm{Na}_{11}^{+}$, and $\mathrm{Na}_{15}^{+}$. Following this scheme, the IRQM excitations in Fig. 3 can be unambiguously identified as particular $1 \mathrm{eh}$ states. They include $\{[101]-[110]\}_{21}$ in $\mathrm{Na}_{7}^{+},\{[220]-$ $[200]\}_{20},\{[220]-[211]\}_{21},\{[220]-[202]\}_{22}$ in $\mathrm{Na}_{11}^{+}$, and $\{[220]-[200]\}_{20},\{[211]-[202]\}_{21},\{[211]-[200]\}_{21}$, $\{[220]-[202]\}_{22}$ in $\mathrm{Na}_{15}^{+}$.

Electric and magnetic modes with the same $\Lambda^{\pi}$ are known to mix in deformed systems (see, e.g., [31]). The mixture of electric E21 and magnetic orbital M11 excitations is especially interesting since it provides access to the orbital M1 scissors mode (SM) 9, 10, 11, 12]. The properties of this mode are sketched in Appendix B.

\section{RESULTS AND DISCUSSION}

A variety of IRQM spectral distributions is shown in Fig. 5. The first line of the figure contains the quadrupole photoabsorption for IRQM states with $\lambda \mu=20,21$ and 22 . Though IRQM cannot be observed in photoabsorption, the latter is useful in any analysis of electron modes and so is worth to be considered. The second line exhibits magnetic dipole strengths for the magnetic dipole scissors mode (SM). The next two lines show the twophoton populations (1) when the TPP runs separately through $\lambda \mu=10$ and 11 branches of the dipole plasmon. The intermediate states in the two-photon process involve all the RPA dipole states from the plasmon region.

The quadrupole spectra in Fig. 5 can be easily identified by 1eh transitions exhibited in Fig. 4. Most of the transitions connect the levels arising due to deformation splitting. The corresponding IRQM are determined by the deformation and vanish at the spherical shape.

The first panel of Fig. 5 shows that IRQM are selectively active in the quadrupole photoabsorption. Some modes $\left(\lambda \mu=22\right.$ in $\mathrm{Na}_{11}^{+}$and $\lambda \mu=20$ and 22 in $\left.\mathrm{Na}_{15}^{+}\right)$ have small E2-transition matrix elements and so are suppressed in this one-photon process. However, as is shown below, these modes can be detected in TPP.

The calculations show that all the IRQM are almost pure 1eh states, i.e. are dominated by one 1eh component (with the related transition in Fig. 4. The contributions of the dominant components typically attain $99-100 \%$. Even in $\lambda \mu=21$ states in $\mathrm{Na}_{15}^{+}$, which most deviate from the $1 e h$ nature, the contribution of the leading 1eh component are $92-93 \%$. This feature is additionally illustrated in Fig. 6 where the unperturbed (the dominant 1eh configuration alone) and RPA IRQM strengths are compared. One sees that in both cases IRQM spectra and strengths are very similar. Only the $\lambda \mu=21$ states in $\mathrm{Na}_{15}^{+}$show considerable redistribution of the strength (keeping, nevertheless, predominantly 1eh structure). This case anticipates the involved picture for heavier clusters where the collective redistribution of strength is much stronger.

The second panel in Fig. 5 exhibits the photoabsorption cross section for the dipole magnetic SM. The one-toone correspondence between E21 and M11 peaks demonstrates the intimate connection between these two modes. As is discussed in Appendix B, the scissors mode is driven by cluster deformation and vanishes in the spherical case. Two $\lambda \mu=21$ states in $\mathrm{Na}_{15}^{+}$represent an instructive example. The lower $\lambda \mu=21$ state determined by $[211] \rightarrow[202]$ transition between the members of the deformation multiplet exhibits an appreciable magnetic dipole strength and thus should carry a large SM fraction. Instead, the higher $\lambda \mu=21$ state is determined by a $[211] \rightarrow[200]$ transition which takes place even in the spherical case. The deformation is not crucial here. This state favors the E21 field and so can be treated as an ordinary quadrupole mode. Altogether, it is seen that deformation-induced IRQM provide access to SM.

The third and fourth panels in Fig. 5 show the TPP cross-section for the reaction paths via $\lambda \mu=10$ and 11 branches of the dipole plasmon. It is seen that TPP response considerably deviates from the photoabsorption. Besides, the population of IRQM crucially depends on the reaction path. For example, it is negligible for $\lambda \mu=21$ states in $\mathrm{Na}_{7}^{+}$(path 10) and $\mathrm{Na}_{15}^{+}$(path 11). Suppression of the TPP transfer in these cases is explained by destructive effects in the photoemission dipole matrix element (see discussion in Appendix A). This matrix element can vary to a large extent, depending on the particular struc- 
ture of IRQM and dipole intermediate states. So, for the efficient population of the particular IRQM, one has to choose the most optimal TPP path. The microscopic calculations can be used here as a guide.

Because of the 1eh nature of IRQM and their strong dependence on cluster deformation, they can deliver a valuable spectroscopic information on the electron levels in the HOMO-LUMO region as well as on the deformation effects in the cluster mean field. For example, the measurement for $\lambda \mu=21$ state in $\mathrm{Na}_{7}^{+}$can provide the energy difference between the Fermi [101] and particle [110] levels (see Fig. 4). If to deduce the Fermi level energy from the ionization potential data, one finally gets the energy of [110] level. Simultaneously we will estimate the deformation splitting of the active subshell. The analysis will be even more effective, if to combine the TPP data with the photoemission and inverse photoemission results for the electron spectra.

\section{EXPERIMENTAL ACCESS TO IRQM}

\section{A. General view}

Two-photon processes are widely used in atomic and molecular physics (for a comprehensive discussion see 32]). Since atomic clusters are similar to molecules, it would be natural to use the same reactions in clusters. However, applications of TPP to clusters are very scarce. This can be partly explained by our poor knowledge on non-dipole low-energy electron modes in clusters, partly by peculiarities of clusters. In this section we will discuss applicability of traditional TPP methods to clusters with a particular accent to IRQM.

As compared with atoms and molecules, atomic clusters have at least three features essential for TPP. i) The dominant decay channel in clusters is usually not radiative. Instead, the levels mainly decay through Landau fragmentation (dissipation of the collective motion through surrounding $1 \mathrm{eh}$ excitation), electron-electron collisions, and electron-ion coupling. This property spoils the radiation-based methods (typical for atomic and molecular measurements) to detect population of levels in clusters. In this connection, we will propose an alternative detection method based on the photoabsorption depletion of cluster beam, which is more suitable for clusters. ii) Cluster excitations have extremely short lifetimes (10-20 fs for the dipole plasmon in medium clusters) and usually are rather broad (see e.g. Fig. 1 for the dipole plasmon). The short lifetimes inhibit the application of adiabatic transfer methods while large widths impede maintaining the resonance conditions. iii) Clusters demonstrate a strong shape isomerism. Cluster beams are usually a statistical mix of clusters of a different shape. This blurs the measured low-energy electron spectra.

Light clusters with their dilute spectrum allow to circumvent some of these troubles. Indeed, Landau frag- mentation in such clusters is very weak, the levels not so broad and the lifetimes are much longer. Light clusters are strictly dominated by one equilibrium shape and their isomeric shapes are close to the ground state one 22. So, we do not expect a strong blurring of the low-energy spectra. Beams of size-selected singly-charged light clusters are readily available. The light deformed singly-charged clusters considered in this paper seem to be most suitable for TPP measurements. The cluster temperature $\sim$ $100 \mathrm{~K}$ could be optimal. Then the thermal broadening of electron levels is small and, at the same time, the effects of the electron-ion coupling are yet smoothed.

In the next subsections we will consider some typical two-photon processes (RS, SEP, STIRAP) and estimate their ability to observe IRQM.

\section{B. Raman scattering}

RS is one of the simplest TPP. In this reaction, a dipole laser-induced transition to an intermediate electronic level (real or virtual) is followed by the dipole fluorescence to low-energy levels.

RS measurements of electron infrared modes in clusters are very rare. We know only one experiment where IRQM were observed in heavy silver clusters embedded into amorphous silica films [19]. The dipole plasmon was used as a resonant intermediate state. The further measurements revealed that IRQM are affected by cluster deformation [36]. These observations show that the radiative decay of the dipole plasmon is detectable in spite of the strong competition with other decay channels. This message is very encouraging for application of TPP to clusters.

RS generally assumes that the coupling between the intermediate dipole and final quadrupole states is of the same order of magnitude as the coupling between the intermediate and ground states. Only then the final state is successfully populated. This means that the absorption and emission dipole matrix elements defined in (A3) and (A5) should be of the same scale. The calculations show that this is indeed the case: in the clusters considered in the present paper most of the absorption and emission dipole matrix elements lie inside the interval 2-10 $e a_{0}$ (in atomic units), i.e. are basically of the same order of magnitude (for exception of some emission matrix elements strictly suppressed due to destructive contributions, see discussion in Appendix A. This means that IRQM in light deformed clusters have a chance to be observed in the resonant RS (running via the dipole plasmon).

RS is effective only if the coupling between intermediate and desirable target levels is strong enough. As was shown for $\mathrm{Na}_{7}^{+}$, this is not always the case. To overcome this trouble, we should consider the methods with stimulated emission into a specific target level (SEP, STIRAP). 


\section{Stimulated emission pumping}

Unlike RS, this method exploits two lasers, pump and dump 33. The pump laser is responsible for the first photoabsorption step. The dump pulse follows the pump one with some delay and couples intermediate to target states. If the difference between the pump and dump frequencies is in resonance with the frequency of the target state, then the dump radiation stimulates the emission to this state.

SEP enjoys widespread application for atoms and molecules but can encounter troubles for clusters where SEP methods to detect the population (based on measurement of spontaneous photoemission or photoelectron yield from certain levels excited by probe lasers) can fail for short-lived cluster levels with their small radiative width. In this connection, we propose a new detection method which does not need any probe radiative procedure.

The idea is to use depletion of cluster beam caused by the photoabsorption. It is known that photoabsorption heats the clusters leading to subsequent evaporation of atoms. The recoil effect from the evaporated atom forces the cluster to leave the beam. Thus the photoabsorption can be measured in terms of depletion. In the scheme we propose, the frequency of the pump laser is fixed in resonance with the dipole plasmon while the frequency of the dump laser scans to get the resonance with IRQM at $\omega_{P}-\omega_{D}=\omega_{I R Q M}$. Then one can detect the IRQM population as a dark resonance in the beam depletion. Indeed if the dump pulse is not in the resonance with IRQM, then a usual photoabsorption induced by the pump laser and additionally supported by the dump laser takes place. In the case of the resonance with IRQM, part of the energy will be used not for cluster heating but for stimulated radiative decay from the dipole plasmon to the given IRQM. So, the photoabsorption (depletion) should demonstrate a sharp decrease, kind of a dark resonance. The deeper the dark resonance, the more populated is the IRQM. The photoionization channel plays no role because the ionization potential in light clusters is higher than $\omega_{P}+\omega_{D}$. If the laser intensities are not too high, then multi-photon ionization can be also neglected. The scheme is simple and suitable for clusters.

However, even in the best case, SEP can transfer to the target level only $25 \%$ of the population [33]. Spontaneous emission from the intermediate level results in an additional leaking. So, it is worth to continue our discussion and consider more efficient methods.

\section{STIRAP}

Much better results can be obtained with STIRAP 34 , 35] which promises up to $100 \%$ of the population transfer from the initial to the target level. So high effectivity is provided by the coherent character of the process and the principle possibility to avoid the leaking from the intermediate levels. Like the previous method, STIRAP also implements two lasers: pump laser to couple the initial state $\mid 0>$ and intermediate state $\mid 1>$ and Stokes laser to stimulate the emission from $\mid 1>$ to the target state $\mid 2>$. However, STIRAP is more involved because it implies the coherent adiabatic transfer.

STIRAP has three principle requirements: i) tworesonance condition $\omega_{P}-\omega_{S}=\omega_{2}-\omega_{0}$ which allows a detuning $\Delta=\left(\omega_{1}-\omega_{0}\right)-\omega_{P}=\left(\omega_{1}-\omega_{2}\right)-\omega_{S}$ from the intermediate state frequency; ii) counterintuitive sequence of the pulses when the Stokes pulse proceeds the pump one; 3) adiabatic evolution.

Under these conditions, one of the time-dependent eigenfunctions of the system is a superposition of initial and target bare states only: $\left|b_{0}(t)>=c_{0}(t)\right| 0>$ $+c_{2}(t) \mid 2>$. It is reduced to $\mid 0>$ at the beginning of the adiabatic evolution and to $\mid 2>$ at the end. Hence, the system finally finds itself in the target state. The intermediate state $\mid 1>$ is not involved to $\mid b_{0}(t)>$ and so is not populated at any time. Thus any leak in the population is avoided and the transfer is complete. The main point is to evolve the system adiabatically, keeping it all the time in the state $\mid b_{0}(t)>$. STIRAP is widely used in atomic and molecular spectroscopy. It is rather insensitive to precise pulse characteristics. Both continuous and pulse lasers can be implemented.

Let's examine the STIRAP requirements for atomic clusters, in particular for the IRQM population via the dipole plasmon.

Two-resonance condition. This condition can be obviously maintained even for a broad dipole plasmon. However, the role of detuning $\Delta$ (which is used as an additional tool to prevent the $\mid 1>$ admixture to $\mid b_{0}(t)>$ ) becomes vague. The dipole plasmon vanishes slowly at its flanks and so rather large detuning is necessary. This can be done even under the restriction $\Delta \ll \omega_{P} \pm \omega_{S}$ imposed by the rotation wave approximation. At the same time, this point was not yet properly investigated and, probably, some fraction of $\mid 1>$ will admix $\mid b_{0}(t)>$. Then the population will not be complete, although it still can remain large.

Counterintuitive order of pump and Stokes pulses. The order when Stokes pulse precedes the pump one is crucial for the maximal population $\underline{34}$, , 35]. Then the Stokes pulse prepares the coherent superposition of the intermediate and final states just before arriving the pump pulse to stimulate the subsequent desirable transfer. Stokes and pump pulses must overlap and the overlapping time $\Delta \tau$ determines duration of the adiabatic evolution. This time should not be longer than the lifetime of the dipole plasmon, i.e. should not exceed 10-100 fs. Though such $\Delta \tau$ is extremely short, it is quite accessible in modern experiments 33].

Adiabatic passage. This condition is most tough. Following [34, 35] it can be formulated as

$$
\Omega \Delta \tau>10
$$

where $\Omega=\sqrt{\Omega_{P}^{2}+\Omega_{S}^{2}}$ is the average of the pump and 
Stokes Rabi frequencies. The Rabi frequency is [34]

$$
\Omega=\frac{|d|}{\hbar} \sqrt{\frac{2 I}{c \epsilon_{0}}} \simeq 2.20 \cdot 10^{8}\left|d\left[e a_{0}\right]\right| \sqrt{I\left[\frac{W}{c m^{2}}\right]} s^{-1},
$$

where $d$ is the dipole coupling matrix element in atomic units, $I$ is the laser intensity in $W / \mathrm{cm}^{2}, c$ is the light speed, $\epsilon_{0}$ is the vacuum dielectric constant. It is easy to estimate (for $d \sim 5 e a_{0}$ and $\Delta \tau=10 \mathrm{fs}$ ) that the condition (2) is maintained for intensities $I>10^{12} \mathrm{~W} / \mathrm{cm}^{2}$. At so high intensities, the multiphoton ionization and fragmentation of clusters should take place [13], which can spoil STIRAP.

However, there is a way to circumvent the trouble. The condition (2) was obtained for one intermediate level. But the realistic spectrum of the dipole plasmon consists of a sequence of dipole levels. In this case, the STIRAP condition should be revised. The realistic case is more complicated but, at the same time, opens new possibilities for STIRAP. In particular, one may loose the adiabatic STIRAP condition and thus the requirements for the laser intensity.

A general case of $\mathrm{N}$ intermediate states, each with its own coupling and detuning was studied in [37]. It was shown that the trapped adiabatic state $\mid b_{0}(t)>$ can be created only when the ratio between each pump coupling and the respective Stokes coupling is the same for all intermediate states. Following our calculations, this condition is unrealistic for atomic clusters. However, the softer alternative adiabatic requirements can be formulated. In particular, in the general case of arbitrary couplings, one may tune the pump and Stokes lasers just below all intermediate states and thus form so called adiabatic-transfer state which also results in a high if not complete population of the target level. Unlike $\mid b_{0}(t)>$, the adiabatictransfer state can have admixtures from the intermediate states during the evolution period $\Delta \tau$ and so some population leaking is unavoidable. Nevertheless, we have here a solid adiabatic transfer with a high population of the target state. Finally, one may conclude that STIRAP can be used for the efficient population of IRQM in clusters.

\section{CONCLUSIONS}

We have presented a first exploration of infrared quadrupole electron modes (IRQM) in light deformed clusters. Most of IRQM are induced by cluster deformation and thus can deliver useful information on deformation effects, e.g. on the deformation splitting of electron levels. Besides, IRQM are about pure one-electron-onehole excitations and so give access to the single-electron spectrum. We explained the origin of IRQM and showed that they can be easily identified in the dilute spectrum of light clusters.

In the second part, we examined some typical twophoton processes (Raman scattering, stimulated emission population and stimulated adiabatic Raman passage) which are widely used in atomic and molecular spectroscopy but not yet for atomic clusters. It was shown that, in spite of some peculiarities of clusters (broad resonances, short level lifetimes, domination of non-radiative decay channels), these TPP can be applied to populate IRQM. Besides, a new method to detect the population of the target cluster states was proposed. TPP measurements of IRQM can be supplemented by photoemission and inverse photoemission experiments delivering the similar information. We hope that our analysis will stimulate application of the experimental TPP methods of atomic and molecular spectroscopy to atomic clusters.

Non-dipole electron excitations in clusters represent a new promising research field. They deliver interesting physics and can serve as a robust test for the theory which still paid main attention to integral characteristics of clusters but not to so fragile patterns as the singleelectron spectra. It worth noting that the single-electron spectra are very sensitive to many cluster features and so can be used as an effective tool for investigation of these features.

Though our calculations have been done for sodium clusters, the qualitative results we obtained are of a general nature and should be valid for other metal clusters as well. In particular, similar deformation-induced IRQM are expected for supported clusters where they can serve as sensitive indicators of the interface interaction and cluster deformation. Besides, IRQM can be important for the quantum transport in clusters where they can lead to a resonant transmission.

\section{Acknowledgments}

The work was supported by the Visitors Program of Max Planck Institute for the Physics of Complex Systems (Dresden, Germany). We thank professors E. Duval and J.-M. Rost for valuable discussions.

\section{APPENDIX A: DIPOLE MATRIX ELEMENTS}

The dipole and quadrupole electron states are described as RPA modes

$$
Q_{\lambda \mu i}^{\dagger}=\frac{1}{2} \sum_{e h}\left(\psi_{e h}^{\lambda \mu i} a_{e}^{\dagger} a_{h}-\phi_{e h}^{\lambda \mu i} a_{e} a_{h}^{\dagger}\right)
$$

where

$$
\psi_{e h}^{\lambda \mu i} \sim N_{\lambda \mu i} \frac{f_{e h}^{\lambda \mu}}{\epsilon_{e h}-\omega_{\lambda \mu i}}, \quad \phi_{e h}^{\lambda \mu i} \sim N_{\lambda \mu i} \frac{f_{e h}^{\lambda \mu}}{\epsilon_{e h}+\omega_{\lambda \mu i}}
$$

are forward and backward amplitudes characterizing contributions of electron-hole (1eh) configurations to the mode. Furthermore, $N_{\lambda \mu i}$ is the normalization coefficient, $f_{e h}^{\lambda \mu}$ is the single-particle matrix element of the residual two-body interaction, $\epsilon_{e h}$ and $\omega_{\lambda \mu i}$ are energies of the 1eh and RPA excitations, respectively. RPA 
describes equally well both collective and non-collective modes. In the latter case (i.e. in the limit $\mid \lambda \mu i>\rightarrow$ $\mid 1 e h>)$, only the amplitude $\left|\psi_{e h}^{\lambda \mu i}\right| \rightarrow 1$ survives while all others vanish.

The photoabsorption matrix element responsible for the coupling between the ground and dipole states is determined by $1 \mathrm{eh}$ part of the dipole operator and reads

$$
\left\langle 1 \mu_{1} i_{1}\left|\operatorname{er} Y_{1 \mu}\right| 0>=\sum_{e h} f_{e h}^{E 1 \mu_{1}}\left(\psi_{e h}^{1 \mu_{1} i_{1}}+\phi_{e h}^{1 \mu_{1} i_{1}}\right)\right.
$$

where $f_{e h}^{E 1 \mu_{1}}$ is the matrix element of the dipole transition. If to put $f_{e h}^{1 \mu_{1}} \approx f_{e h}^{E 1 \mu_{1}}$ and to take into account that all the relevant dipole $1 e h$-levels are blue-shifted by the dipole interaction, then

$$
\left\langle 1 \mu_{1} i_{1}\left|\operatorname{er} Y_{1 \mu}\right| 0>\approx 2 N_{1 \mu_{1} i_{1}} \sum_{e h} \frac{\left(f_{e h}^{E 1 \mu_{1}}\right)^{2} \epsilon_{e h}}{\epsilon_{e h}^{2}-\omega_{1 \mu_{1} i_{1}}^{2}}\right.
$$

and it is easy to see that all $1 e h$ levels contribute constructively to the transition.

Instead, the photoemission dipole matrix element connecting the dipole and quadrupole RPA modes is determined by 1ee and 1 hh parts of the dipole operator and has the more complicated structure:

$$
\begin{array}{r}
\left\langle 2 \mu_{2} i_{2}\left|e r Y_{1 \mu}\right| 1 \mu_{1} i_{1}\right\rangle \\
=\frac{1}{2}\left[\sum_{e e^{\prime}} f_{e e^{\prime}}^{E 1 \mu} \sum_{h}\left\langle\psi_{e h}^{2 \mu_{2} i_{2}} \psi_{h e^{\prime}}^{1 \mu_{1} i_{1}}+\psi_{h e^{\prime}}^{2 \mu_{2} i_{2}} \psi_{h e}^{1 \mu_{1} i_{1}}\right\rangle\right. \\
\left.+\sum_{h h^{\prime}} f_{h h^{\prime}}^{E 1 \mu} \sum_{e}\left\langle\psi_{h e}^{2 \mu_{2} i_{2}} \psi_{e h^{\prime}}^{1 \mu_{1} i_{1}}+\psi_{h^{\prime} e}^{2 \mu_{2} i_{2}} \psi_{e h}^{1 \mu_{1} i_{1}}\right\rangle\right] .
\end{array}
$$

The terms with backward amplitudes, $\sim \phi \phi$, are omitted in A5 since they are usually small.

The matrix element A5 becomes simpler when the quadrupole state is dominated by a single $1 e h$ configuration, say $\left\{\bar{e}_{2} \bar{h}_{2}\right\}$. As is shown in Sec. IV] this is indeed a common case. Then all the quadrupole amplitudes vanish except of $\left|\psi_{\bar{e}}^{2 \mu_{2} i_{2}}\right| \rightarrow 1$ and Eq. (A5) is reduced to

$$
\begin{array}{r}
\left\langle\left\{\bar{e}_{2} \bar{h}_{2}\right\}\left|e r Y_{1 \mu}\right| 1 \mu_{1} i_{1}\right\rangle \\
= \pm \frac{1}{2}\left[\sum_{e} f_{\bar{e}_{2} e}^{E 1 \mu_{1}} \psi_{\bar{h}_{2} e}^{1 \mu_{1} i_{1}}+\sum_{h} f_{h \bar{h}_{2}}^{E 1 \mu} \psi_{\bar{e}_{2} h}^{1 \mu_{1} i_{1}}\right] .
\end{array}
$$

Now only the dipole amplitudes including the hole (first term) or particle (second term) from the pair $\left\{\bar{e}_{2} \bar{h}_{2}\right\}$ contribute to the transition. The contribution is considerable only subject to the large dipole amplitude and strong matrix element. (The latter take place if the matrix element fulfills the asymptotic Nilsson selection rules [27, 28]: E1 $\mu$ transition between $\left[\mathcal{N}_{i} n_{i z} \Lambda_{i}\right]$ and $\left[\mathcal{N}_{j} n_{j z} \Lambda_{j}\right]$ states is favored if $\mathcal{N}_{i}=\mathcal{N}_{j} \pm 1, \quad n_{i z}=n_{j z} \pm 1$ for $\mu=0$ and $n_{i z}=n_{j z}$ for $\mu=1$ ). Under these tough requirements, only a few terms yield large contributions to (A6). Depending on the structure of the dipole and quadrupole states, the contributions can have different signs and thus lead to constructive or destructive results. So, the magnitude of the photoemission matrix element (and thus the coupling between the dipole and quadrupole states) can be large (like for the photoabsorption) or very small.

\section{APPENDIX B: SCISSORS MODE}

The SM is a general dynamical phenomenon already found or predicted in different finite quantum systems (atomic nuclei, metal clusters, quantum dots, dilute ultra-cold gases of Bose and Fermi atoms), see the review [12]. All these different systems have two features in common: broken spherical symmetry (deformation) and a two-component structure.

Like the quadrupole modes, the SM separates into lowenergy $(\Delta \mathcal{N}=0)$ and high-energy $(\Delta \mathcal{N}=2)$ branches. In this paper we consider only the low-energy branch which is mixed with IRQM. Macroscopically, this branch is treated as a small-amplitude rotational oscillations of a spheroid of valence electrons against a spheroid of the ionic background (hence the name scissors mode). Like IRQM, the SM is driven by cluster deformation and its energy scale is naturally determined by the deformation splitting of the electron levels. In axial clusters with a quadrupole deformation $\delta_{2}$, the energy and magnetic strength of the mode are estimated as [9, 10]:

$$
\omega=\frac{20.7}{r_{s}^{2}} N_{e}^{-1 / 3} \delta_{2} \mathrm{eV}, \quad B(M 1) \simeq N_{e}^{4 / 3} \delta_{2} \mu_{b}^{2}
$$

where $r_{s}$ is the Wigner-Seitz radius (in $\AA$ ), $N_{e}$ is the number of valence electrons, and $\mu_{b}$ is the Bohr magneton. It is seen that both the energy and strength are proportional to the deformation parameter $\delta_{2}$ and vanish for the spherical shape.

In axially symmetric systems, the SM is generated by the orbital momentum fields, $L_{x}$ and $L_{y}$, perpendicular to the symmetry axis $z$ and, like the quadrupole mode $\lambda \mu=21$, forms the states $\mid \Lambda^{\pi}=1^{+}>$. SM strongly responds to an external magnetic dipole field. Besides, it determines the van Vleck paramagnetism in deformed clusters 19, 10, 11, 12]. The experimental search of the SM in clusters is still in very beginning [19].

There is an intimate connection between the scissors and quadrupole E21 modes in deformed clusters. If fact, both SM and E21 mode are parts of a general motion of multipolarity $\lambda \mu=21$. To illustrate this point, we expand a single-particle electron state $\nu$ in terms of a spherical basis $(n L \Lambda)$

$$
\Psi_{\nu=\left[\mathcal{N} n_{z} \Lambda\right]}=\sum_{n L} a_{n L}^{\nu} R_{n L}(r) Y_{L \Lambda}(\Omega)
$$

and estimate the SM 1eh matrix element:

$$
\begin{aligned}
\left\langle\Psi_{p}\left|\hat{L}_{x}\right| \Psi_{h}\right\rangle & \propto \delta_{\pi_{p}, \pi_{h}} \delta_{\Lambda_{p}, \Lambda_{h} \pm 1} \\
& \cdot \sum_{n L} a_{n L}^{p} a_{n L}^{h} \sqrt{L(L+1)-\Lambda_{h}\left(\Lambda_{h} \pm 1\right)} .
\end{aligned}
$$

It is seen that the SM operator connects only components from one and the same basis level $n L$. Indeed, the operators $\hat{L}_{x}$ and $\hat{L}_{y}$ have no $r$-dependent part and so, due to orthogonality of the basis functions $R_{n L}(r)$, cannot connect the components with different $n L$. But the 
latter can be done by the quadrupole operator $r^{2} Y_{21}$. In this sense the SM operator is more selective than E21, though both operators generate transitions of the same multipolarity. $\Lambda^{\pi}=1^{+}$states involve both SM and E21 modes and respond to both M11 and E21 external fields. The states are treated as magnetic dipole SM or electric quadrupole E21, depending on each of the two responses dominates.
[1] G. Mie, Ann. Phys. (Leipzig) 25, 377 (1908).

[2] U. Kreibig and M. Vollmer, Optical properties of metal clusters (Springer, Berlin, 1993).

[3] W.A. de Heer, Rev. Mod. Phys. 65, 611 (1993).

[4] M. Brack, Rev. Mod. Phys. 65, 677 (1993).

[5] Clusters of atoms and molecules, ed. H. Haberland, (Springer series in chemical physics, 52, Springer, Berlin, 1994).

[6] V.O. Nesterenko, W. Kleinig, and F.F. de Souza Cruz, in Proc. of Intern. Workshop "Collective excitations in Fermi and Bose Systems", Serra Negra, San Paulo, Brazil, 1998 edited by C.A. Bertulani, L.F. Canto and M.S. Hussein (World Scientific, Singapore, 1999), p. 205224.

[7] G.F. Bertsch and R. Broglia, Oscillations in finite quantum systems, (Cambridge Univ. Press, 1994).

[8] V.O. Nesterenko, P.-G. Reinhard, W. Kleinig, under preparation for publication.

[9] E. Lipparini and S. Stringari, Phys. Rev. Lett. 63, 570 (1989); Z. Phys. D 18, 193 (1991).

[10] V.O. Nesterenko, W. Kleinig, F.F. de Souza Cruz and N. Lo Iudice, Phys. Rev. Lett. 83, 57 (1999).

[11] P.-G. Reinhard, V.O. Nesterenko, E. Suraud, S. El Gammal, and W. Kleinig, Phys. Rev. A 66, 013206 (2002).

[12] V.O. Nesterenko, W. Kleinig, P.-G. Reinhard, N. Lo Iudice, F.F. de Souza Cruz, and J.R. Marinelli, Eur. Phys. J. D27, 43 (2003).

[13] F. Calvayrac, P.-G. Reinhard, E. Suraud, and C.A. Ullrich, Phys. Rep. 337, 493 (2000).

[14] P.-G. Reinhard and E. Suraud, Introduction to Cluster Dynamics, (Wiley-VCH, Berlin, 2003).

[15] W. Ekardt, Phys. Rev. Lett. 52, 1925 (1984).

[16] W. Kleinig, V.O. Nesterenko, P.-G. Reinhard, and Ll. Serra, Eur. Phys. J. D 4, 343 (1998).

[17] W. Kleinig, V.O. Nesterenko, and P.-G. Reinhard, Ann. Phys. (NY) 297, 1 (2002).

[18] V.O. Nesterenko, W. Kleinig, and P.-G. Reinhard, Eur. Phys. J. D 19, 57 (2002).

[19] H. Portales, E. Duval, L. Saviot, M. Fujii, M. Sumitoto, and S. Hayashi, Phys. Rev. B 63, 233402 (2001).

[20] O. Gunnarson and B.I. Lundqvist, Phys. Rev. B 13, 4274 (1976).

[21] B. Montag, Th. Hirschmann, J. Meyer, P.-G. Reinhard, and M Brack, Phys. Rev. B 52, 4775 (1995).

[22] S. Kümmel, M. Brack, and P.-G. Reinhard, Phys. Rev. B62, 7602 (2000).

[23] M. Schmidt and H. Haberland, Eur. Phys. J. D6, 109 (1999).

[24] B. Montag, P.-G. Reinhard, J. Meyer, Z. Phys. D 32, 125 (1994).

[25] M. Weissbluth, Atoms and Molecules (Academic Press, New York, 1978).

[26] K. Clemenger, Phys. Rev. B 32, 1359 (1985).

[27] S.G. Nilsson, K. Dan. Vidensk. Selsk. Mat. Fys. Medd., 29, n. 16 (1955).
[28] A. Bohr and B. Mottelson, Nuclear Structure, v. 2, (Benjamin, Reading, MA, 1975).

[29] P.-G. Reinhard, O. Genzken, and M. Brack, Ann. Phys. (Leipzig) 5, 1 (1996).

[30] L. G. Gerchikov, A. N. Ipatov, A. V. Solov'ev, and W. Greiner, J. Phys. B 31, 3065 (1998).

[31] N. Lo Iudice, Prog. Part. Nucl. Phys. 28, 556 (1997).

[32] Atomic and Molecular Beam Methods, ed. G. Scoles, (Oxford Univ. Press, Oxford, 1988).

[33] Molecular dynamics and spectroscopy by stimulated emission pumping, ed. H.-L. Dai and R.W. Field (Advanced series in physical chemistry, 4, World Scientific, Singapure, 1999).

[34] K. Bergmann and B.W. Shore, in Molecular dynamics and spectroscopy by stimulated emission pumping, ed. H.L. Dai and R.W. Field (Advanced series in physical chemistry, 4, World Scientific, Singapure, 1999), Chapter 9, p. 319.

[35] K. Bergmann, H. Theuer, and B.W. Shore, Rev. Mod. Phys. 70, 1003 (1998).

[36] E. Duval, private communication.

[37] N.V. Vitanov and S. Stenholm, Phys. Rev. A60, 3820 (1999). 


\section{FIGURE CAPTIONS}

Figure 1: Photoabsorption dipole cross section in light axially deformed $\mathrm{Na}$ clusters. Quadrupole and hexadecapole deformation parameters are indicated in boxes. RPA results are given as vertical bars (in $e V \AA^{2}$ ) and as a strength function smoothed by the Lorentz weight with the averaging parameter $0.25 \mathrm{eV}$. Separate contributions to the strength function from the $\lambda \mu=10$ and 11 dipole branches (the latter is twice stronger) are shown by dashed curves. The experimental data (triangles) from 23] are given for the comparison.

Figure 2: Two-photon process: scheme of population of IRQM states $\lambda \mu=20,21$ and 22 via the $\lambda \mu=10$ (left) and 11 (right) branches of the dipole plasmon.

Figure 3: Quadrupole strength distribution in light Na clusters. Lower panels: the unperturbed 1eh strength (without residual interaction). Upper panels: the RPA strength (with residual interaction). The results are given as bars (for every discrete RPA or 1eh state) and as smooth strength functions obtained by folding with a Lorentzian of width $\Delta=0.25 \mathrm{eV}$. The IRQM strength (enclosed by the circles at $0.5-1.5 \mathrm{eV}$ ) is very weak and so rescaled by the factor $10^{2}$.

Figure 4: The electron level scheme for $\mathrm{Na}_{7}^{+}, \mathrm{Na}_{11}^{+}$ and $\mathrm{Na}_{15}^{+}$in the spherical limit (left) and at the equilibrium deformation (right). Occupied and unoccupied states are drawn by solid and dashed lines, respectively. The Fermi (HOMO) level is marked by index F. Arrows depict the possible low-energy hole-electron $E 2 \mu$ transitions.

Figure 5: IRQM in light clusters. The plots exhibit quadrupole photoabsorption (uppermost panels, marked E2), scissors M1 photoabsorption (second line, marked M1), and two-photon population of IRQM via $\lambda \mu=10$ (third line, marked TPP E10) and 11 (lowest panels, marked TPP E11) dipole branches. IRQM are depicted by solid $(\lambda \mu=20)$, dashed $(\lambda \mu=21)$, and dotted $(\lambda \mu=22)$ curves. The strengths are smoothed by a Lorentzian with $\Delta=0.1 \mathrm{eV}$.

Figure 6: Quadrupole strengths for particular IRQM. The unperturbed 1eh (dashed curve) and RPA (solid curve) strengths are compared. The results are smoothed by a Lorentzian with the width $\Delta=0.1 \mathrm{eV}$. 


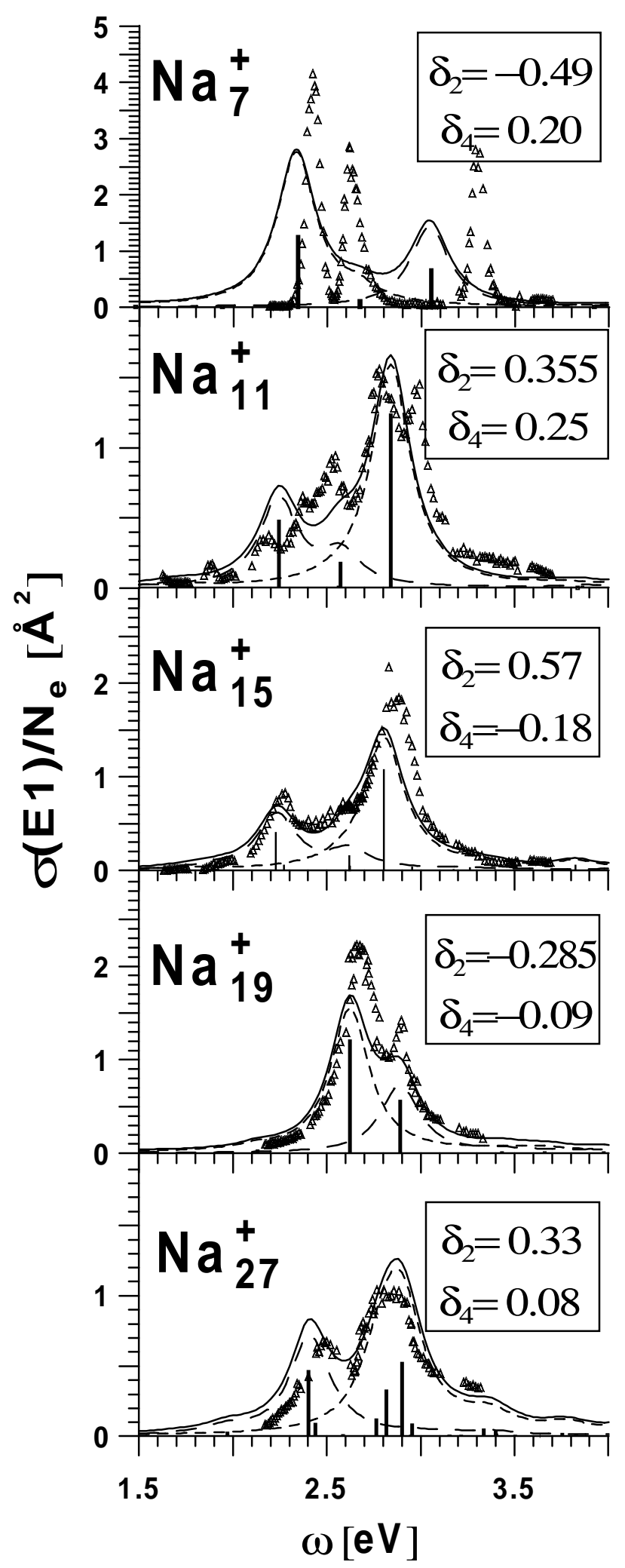




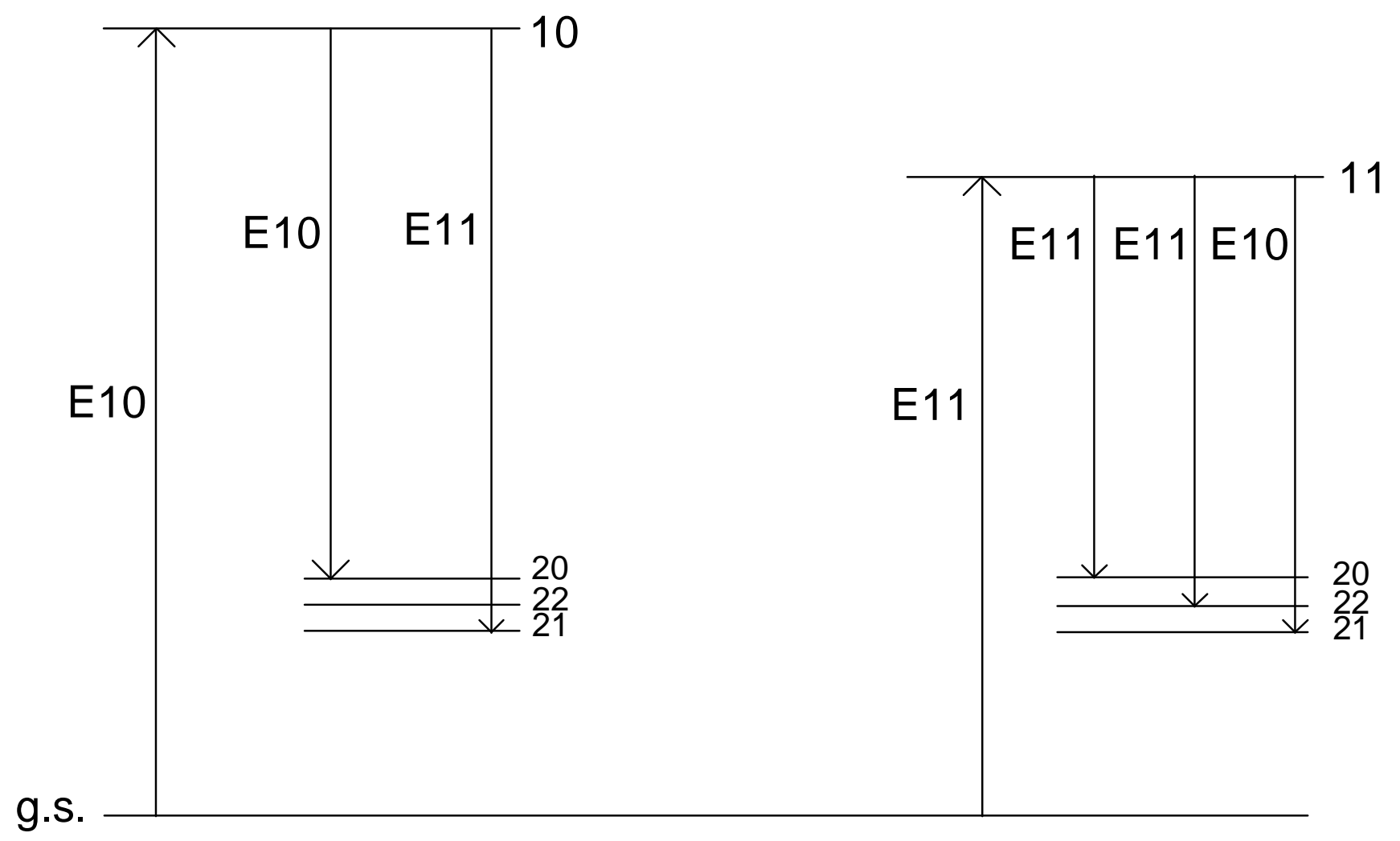



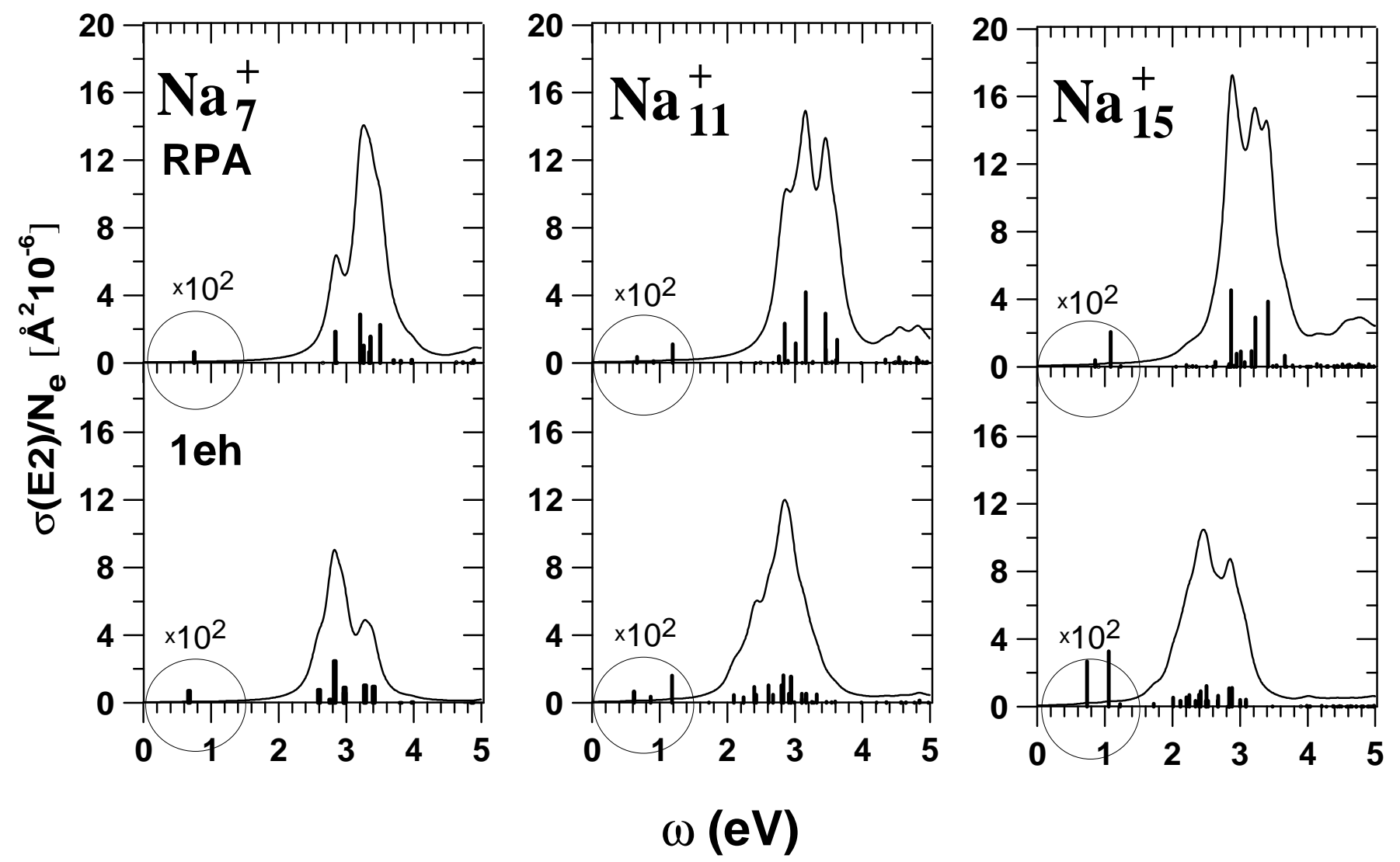

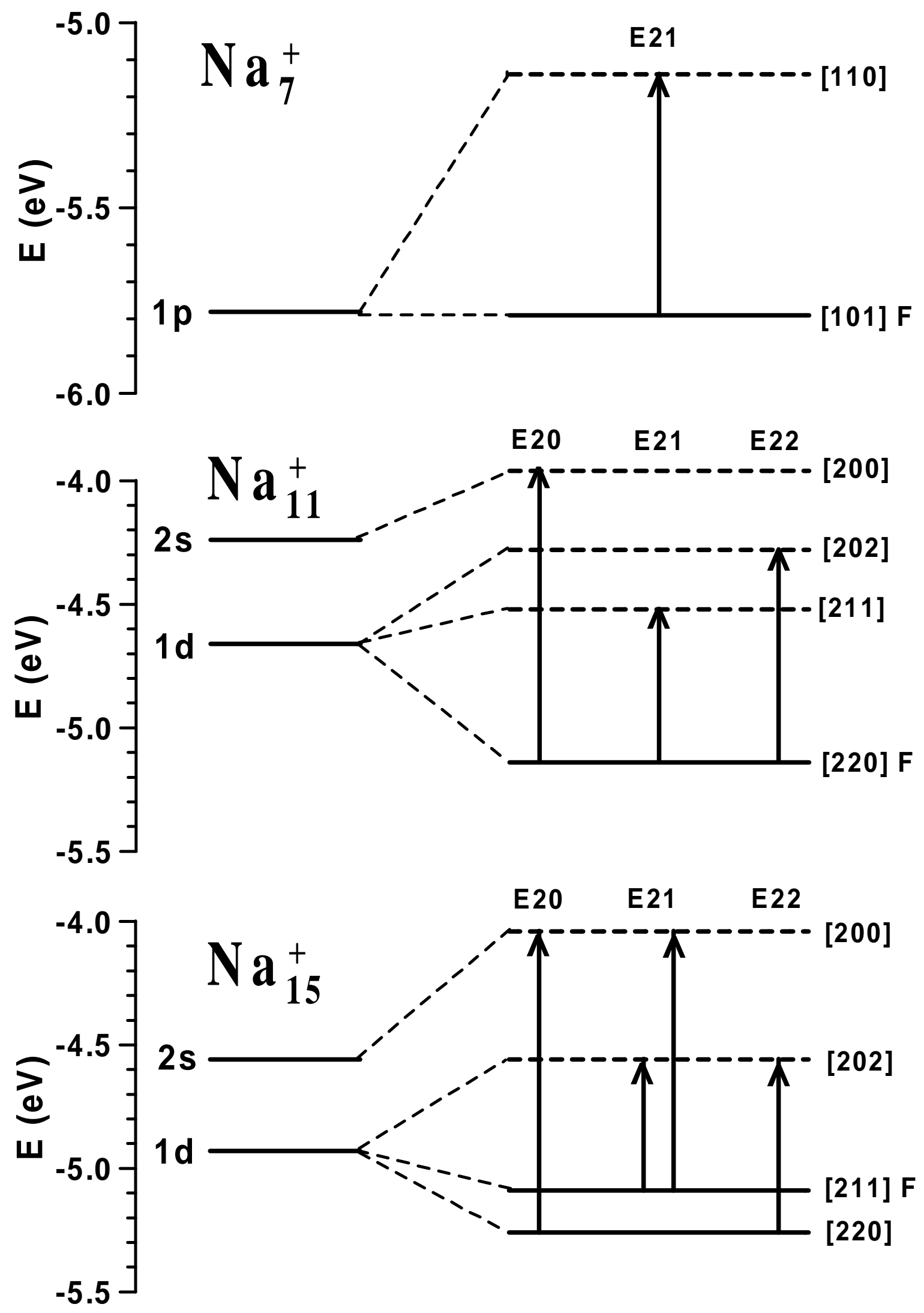

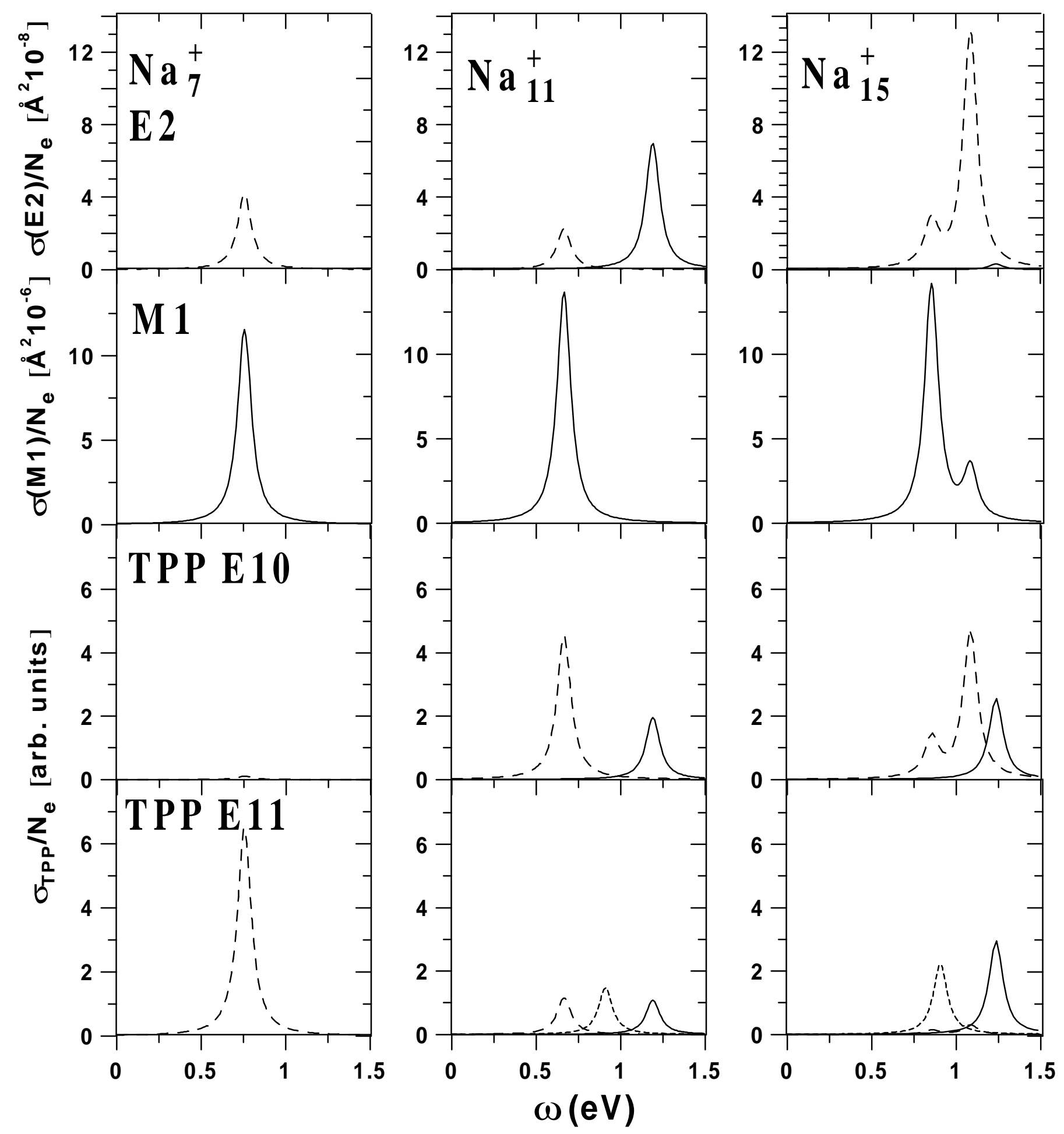


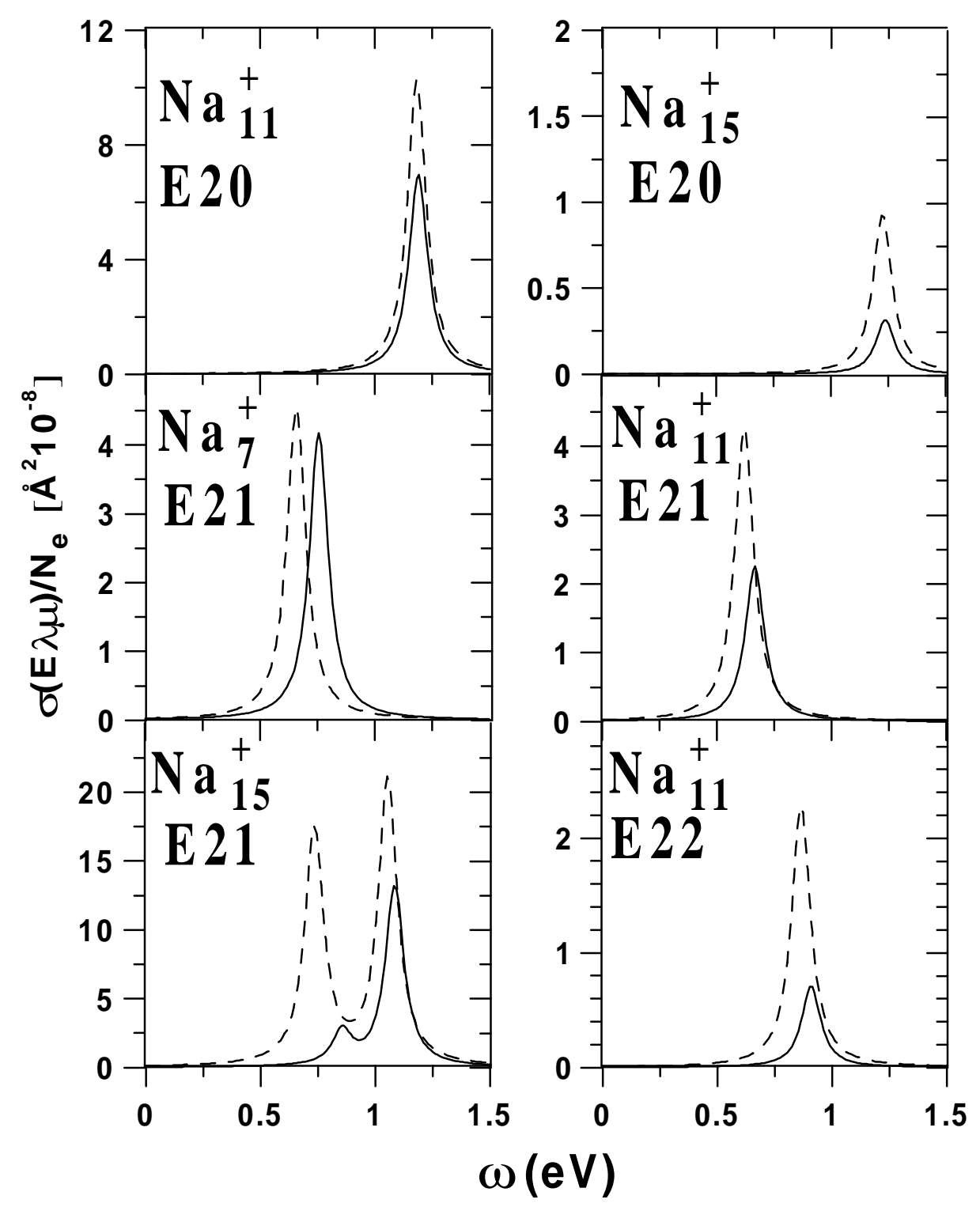

